\title{
Study the effect of alpha particle fluences on the morphology and optical properties of poly-aniline in nano-scale
}

\author{
M. Shaban and M. F. Eissa ${ }^{1}$ \\ Physics Department, Faculty of Science, Beni-Suef University, Beni-Suef, Egypt. \\ 1) Address correspondence to Mostafa Fawzy Eissa, Physics Department, Beni-Suef University, Beni-Suef 62511, \\ Beni-Suef, Egypt.E.mail: mostafa.eisa@science.bsu.edu.eg.
}

\begin{abstract}
Poly-aniline is one of the most important conducting polymers. The poly-aniline has many applications in the electronic fields such as batteries, sensors, controlling systems and organic displays. It is good environmental stability, easy conductivity control and cheap production in large quantities. In this study poly-aniline samples in nan-structure were irradiated with $\alpha$-particles with different fluences $\left(1.16 \times 10^{8}-1.20 x\right.$ $10^{9}$ alphas $\left./ \mathrm{cm}^{2}\right)$ and constant energy $(5.32 \pm 0.23 \mathrm{MeV})$. The damage is almost regular along the path length of alpha particles in poly-aniline samples. The modifications in the morphology and optical properties induced by the radiation were measured. It was found a strong correlation between absorbance and the alpha particle fluences at wavelength $600 \mathrm{~nm}$ for the samples after irradiations. Also, the results showed increase the number of carbon atoms per cluster in the poly-aniline samples after irradiations.
\end{abstract}

Keywords: Poly-aniline, Alpha particles, band gap energy, cluster size

\section{Introduction}

Conducting polymers is a prospective class of new materials that combine solubility, process ability, and flexibility of plastics with electrical and optical properties of metals and semiconductors [1]. These conducting polymers exhibit the unusual electronic properties such as electrical conductivity, low energy optical transitions, low ionization potential and high electron affinity [2] due to the presence of extended $\pi$-conjugated systems along the polymeric chain [3]. Poly-aniline (PANI) is one of the most important conducting polymers because of excellent electrical properties, electrochemical, electro-rheological. These properties found potential applications in the electronic fields such as batteries, sensors, controlling systems and organic displays [4-7] because of its facile synthetic process, good environmental stability, easy conductivity control and cheap production in large quantities. Poly-aniline can be found in one of three idealized oxidation states: leucoemeraldine $\left(\mathrm{C}_{6} \mathrm{H}_{4} \mathrm{NH}\right)_{\mathrm{n}}$, emeraldine $\left(\left\{\left[\mathrm{C}_{6} \mathrm{H}_{4} \mathrm{NH}\right]_{2}\left[\mathrm{C}_{6} \mathrm{H}_{4} \mathrm{~N}\right]_{2}\right\}_{n}\right)$ and pernigraniline $\left(\mathrm{C}_{6} \mathrm{H}_{4} \mathrm{~N}\right)_{\mathrm{n}}$. Emeraldine base is regarded as the most useful form of poly-aniline due to its high stability at room temperature and the fact that, upon doping with acid, the resulting emeraldine salt form of poly-aniline is highly electrically conducting. But leucoemeraldine and pernigraniline are poor conductors, even when doped with an acid [8-9]. When an ionization radiation passes through a polymeric material, ionization and excitation for molecules of the material are produced [10]. These lead to breaking of original bonds, chain scission, radical formation and cross-linking in polymeric material [11-12]. Scission and cross-linking not only depend upon polymer structure but also upon the energy deposited per unit track length (LET) [13-15]. This, in turn, tends to modify the structure and optical properties of polymers [16-17]. Clusters (number of carbon atoms per cluster) be formed along alpha particle tracks. These clusters supposed to be carriers of electrical conductivity in ion irradiated polymers and influence the optical properties [18]. In the present study, the effect of alpha particle fluences $\left(1.16 \times 10^{8}-1.20 \times 10^{9}\right.$ alphas $\left./ \mathrm{cm}^{2}\right)$ and constant energy $(5.32 \pm 0.23 \mathrm{MeV})$ on the structure and optical properties of PANI thin films on glass substrate has been investigated.

\subsection{Production of PANI samples}

\section{Experimental techniques}

A $0.1 \mathrm{M}$ ammonium persulfate (APS) was dissolved in $50 \mathrm{~mL}$ water and kept for $1 \mathrm{~h}$ at room temperature. Also, a $0.1 \mathrm{M}$ aniline monomer was prepared in $50 \mathrm{~mL}$ water and also kept for $1 \mathrm{~h}$ at room temperature. To obtain high yield and massive production, PANI was deposited on glass substrate by rapid mixing of the reactants.

\subsection{Irradiation process}

Holder collimator with a certain height was used to verify normal irradiation for the poly-aniline (1.00 x $1.00 \mathrm{~cm}^{2} \times 20 \mathrm{~nm}$ ) in air by $\alpha$-particles through a collimator holder at Beni-Suef University, Egypt [19]. The density of poly-aniline was $1.245 \mathrm{~g} / \mathrm{cm}^{3}$. The energy of alpha particle that emerged from the collimator was 
determined using a surface barrier detector. The height of the collimator would reduce the energy of $5.486 \mathrm{MeV}$ $\alpha$-particles which emitted from ${ }^{241} \mathrm{Am}$ - source to $5.32 \pm 0.23 \mathrm{MeV}$.

The incident flux $(\phi)$ was calculated by the following equation:

$$
\phi=\frac{A}{4 \pi r^{2}}
$$

Where $\mathrm{A}$ is the activity of the ${ }^{241} \mathrm{Am}$ - source in $\mathrm{Bq}$ and $\mathrm{r}$ is the source-detector distance in centimeters.

The fluence $(\Phi)$, the total number of $\alpha$ - particles emitted from the collimator and incident on CR-39 per unit area in a certain irradiation time $(\mathrm{t})$, is:

$$
\Phi=\varphi t
$$

The irradiations were verified at irradiation times $6.50,41.00$, and $71.00 \mathrm{~h}$. The corresponding fluences according to equation (2) were $1.16 \times 10^{8}, 7.48 \times 10^{8}$ and $1.2 \times 10^{9}$ alphas $/ \mathrm{cm}^{2}$, respectively. The error in the fluence was $1604 \mathrm{alpha} / \mathrm{cm}^{2}$.

\subsection{Morphology measurements}

A field emission scanning electron microscope (FE-SEM), (Model: Leo Supra 55, 1nm resolution, USA) was used to characterize the morphology of the PANI films before and after irradiations.

\subsection{Optical absorption spectra}

The optical spectra of the virgin and irradiated PANI films were measured by using UV-VIS-NIR spectrophotometer (Shimadzu UV-1601 PC, Japan) in the wavelength range of 300-800 nm).

\section{Results and discussion}

When energetic alpha particles penetrate the medium, they create damage along the particle trajectory. According to the SRIM program calculations [20], the projected range of $5.32 \mathrm{MeV}$ alpha particles in PANI samples was calculated to be $33.13 \mu \mathrm{m}$ and the LET was $10.54 \mathrm{eV} / \AA$. The thickness of the PANI samples (20 $\mathrm{nm}$ ) is small compared with the projected range. According to the Bragg curve (the curve consists of a flat region known as the plateau, after which the LET rises up to its peak value in a region called the Bragg peak, the region of maximum energy transfer), LET is in the flat region. Therefore, the damage is almost regular along the trajectory of alpha particles in PANI samples. The overlapping tracks and the density of the damage (track density) may increase with increasing the irradiation time or fluence of alpha particles. These, in turn, change the physical and chemical properties of the PANI samples.

\subsection{PANI morphology}

Figure 1 shows top-view SEM images of PANI thin films of the virgin and irradiated samples with different irradiation times or fluences of alpha particles. Smooth and uniform thin film of virgin PANI sample is deposited on the glass substrate for $20 \mathrm{~min}$, as shown in Figure 1(a). The PANI sample irradiated with alpha particles at $6.50 \mathrm{~h}$ showed some nanoparticles aggregation as shown in Figure 1(b). Formation of clusters on the PANI thin film is noticed at irradiation time $41.00 \mathrm{~h}$, as displayed in Figure 1(c). The density of the formed nanostructures decreases for prolong the irradiation time to $71.00 \mathrm{~h}$, as observed in Figure 1(d). 


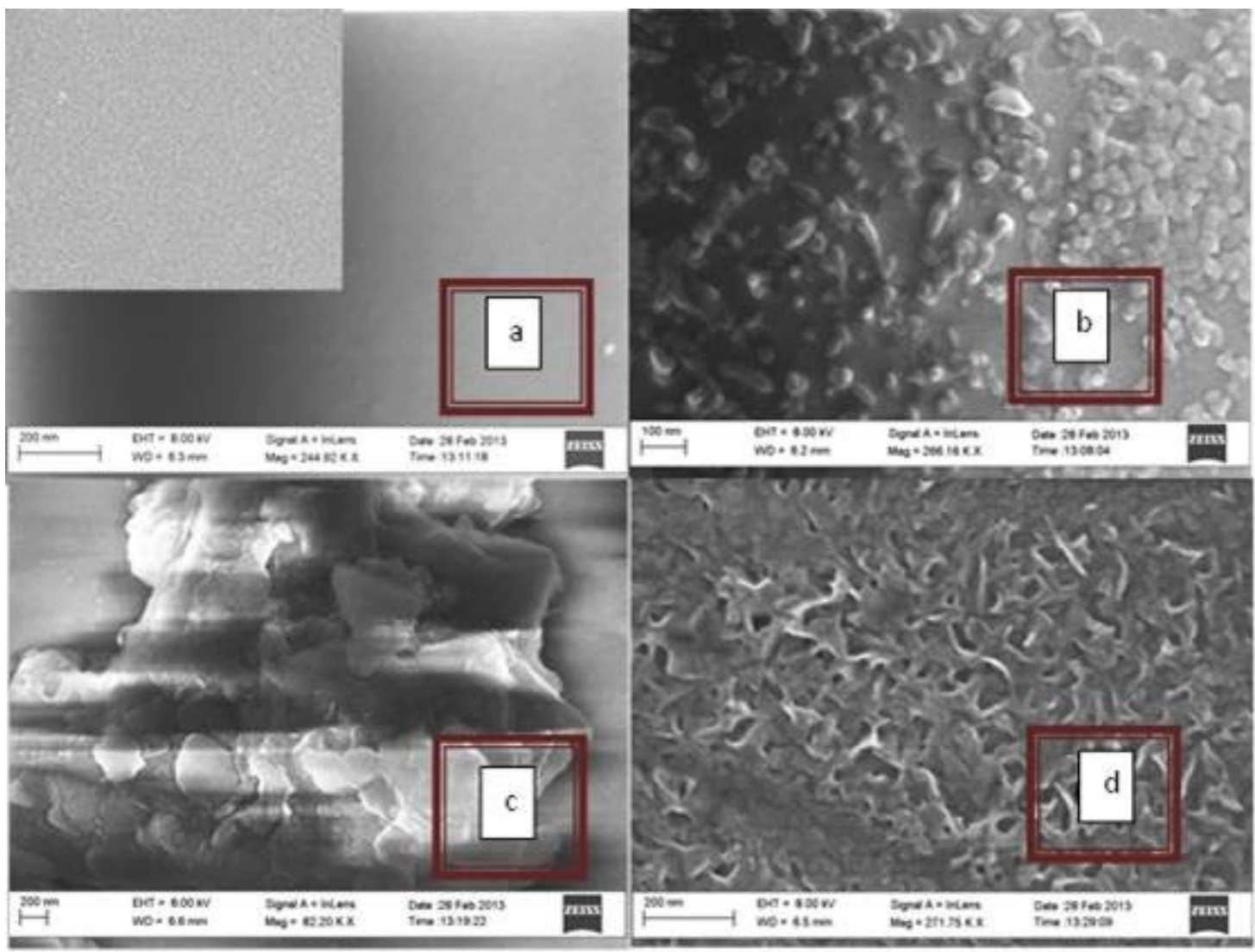

Fig. 1. Top-View FE-SEM images of synthesized PANI samples on glass substrate for virgin and irradiated by alpha particle with different irradiation times: (a) Virgin, (b) $6.50 \mathrm{~h}$, (c) $41.00 \mathrm{~h}$ and (d) $71.00 \mathrm{~h}$.

\subsection{Optical properties}

Irradiation of polymers, undergo main chain scission and cross-linking reactions leading to change the molecular weight products and the surface morphology accompanied with change in optical properties [21]. The optical absorption method can be used for the investigation of the optically induced transitions which can provide information about the band structure and energy gap in crystalline and non-crystalline materials. The UV-VIS spectra of virgin and irradiated PANI samples are shown in Fig. 2. It is clear from the spectra that there is a red shifting in the absorption edge for the irradiated samples from the virgin sample. The red shift indicates a decrease in the band gap after irradiations due to the degradation as a result of bond cleavage in the PANI samples. In addition to, there is a negative correlation between absorbance and the present irradiation times of the alpha particles in the wavelengths range of $530-705 \mathrm{~nm}$. It was found that, the correlation coefficient was 0.99 at wavelength, $600 \mathrm{~nm}$. These reflect that the samples after irradiations with the present alpha particle fluences are sensitive to the visible light at the wavelengths $530-705 \mathrm{~nm}$. 


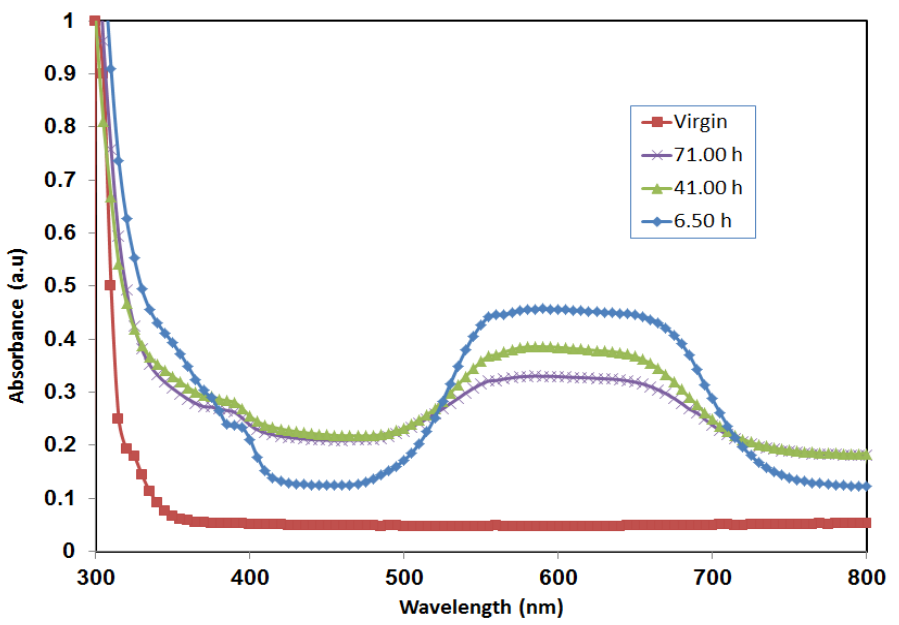

Fig. 2. UV- Vis spectra of the virgin and irradiated of nano-PANI samples with alpha particles at different irradiation times.

The optical band gap energy $\left(E_{g}\right)$ for the PANI samples was calculated from Tauc's expression by the extrapolation of the plot of graph between the photon energy $(\mathrm{h} v)$ and $(\alpha \mathrm{h} v)^{1 / \mathrm{n}}$ for virgin and irradiated PANI samples as shown in Figs.3-6. The Tauc's expression [18] is given by:

$\alpha^{\prime}(v)=\beta\left(h v-E_{g}\right)^{n} / h v$

Where $\beta$ is a constant, $\alpha$ is the absorption coefficient of the photons in the samples and $\mathrm{n}$ is a constant depends on the type of transition. This behavior suggested that the indirect allowed transition $(n=2)$ is the most probable involved transition mechanism. Table I displays the variations of the $\mathrm{E}_{\mathrm{g}}$ for the virgin and irradiated PANI samples with alpha particles of different irradiation times. The present study confirmed that the $\mathrm{E}_{\mathrm{g}}$-value for the virgin sample was reduced due to alpha particle irradiations. The variations in $\mathrm{E}_{\mathrm{g}}$ suggest formation of defects (radicals and organic species) after alpha particles irradiations and/or the formation of carbon enrich clusters [22]. This may be attributed to the formation of a conjugated system of bonds due to bond cleavage by radiation.

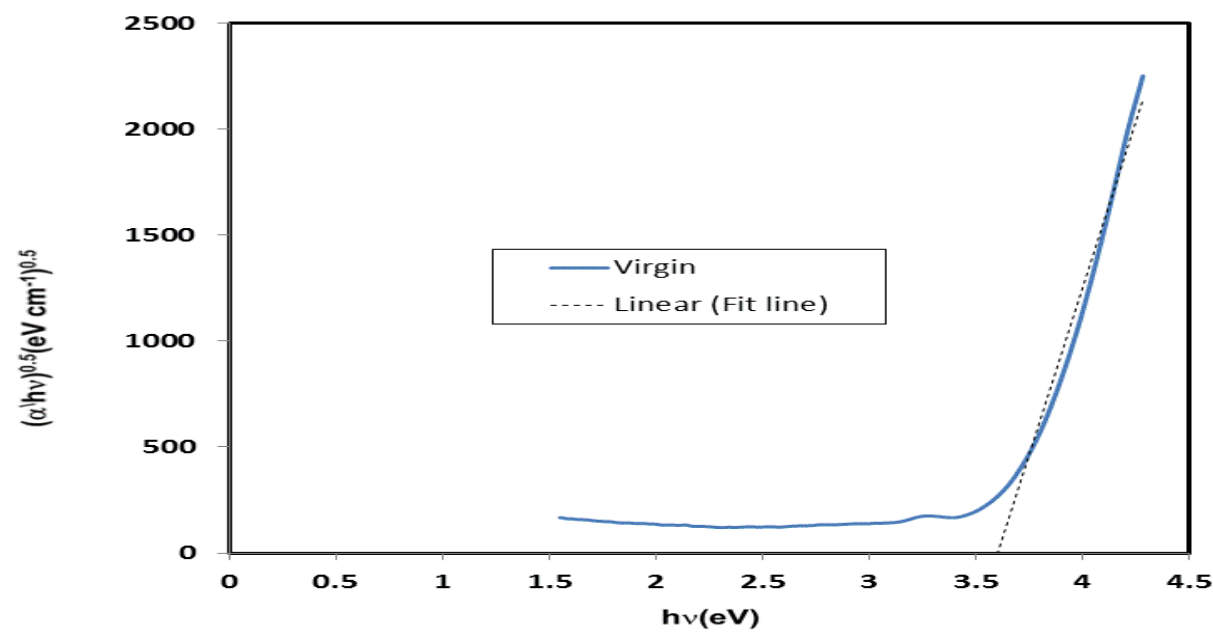

Fig. 3. Tauc's plot corresponding to indirect band gap for virgin PANI sample. 


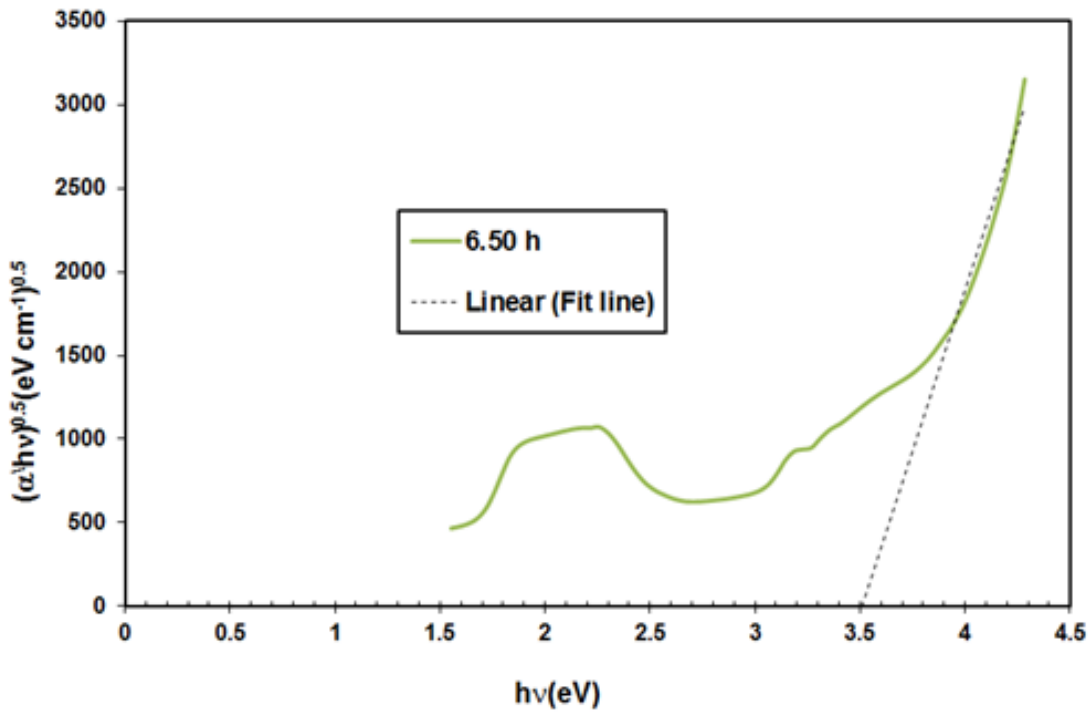

Fig. 4. Tauc`s plot corresponding to indirect band gap for irradiated PANI samples at alpha particle irradiation time $6.50 \mathrm{~h}$

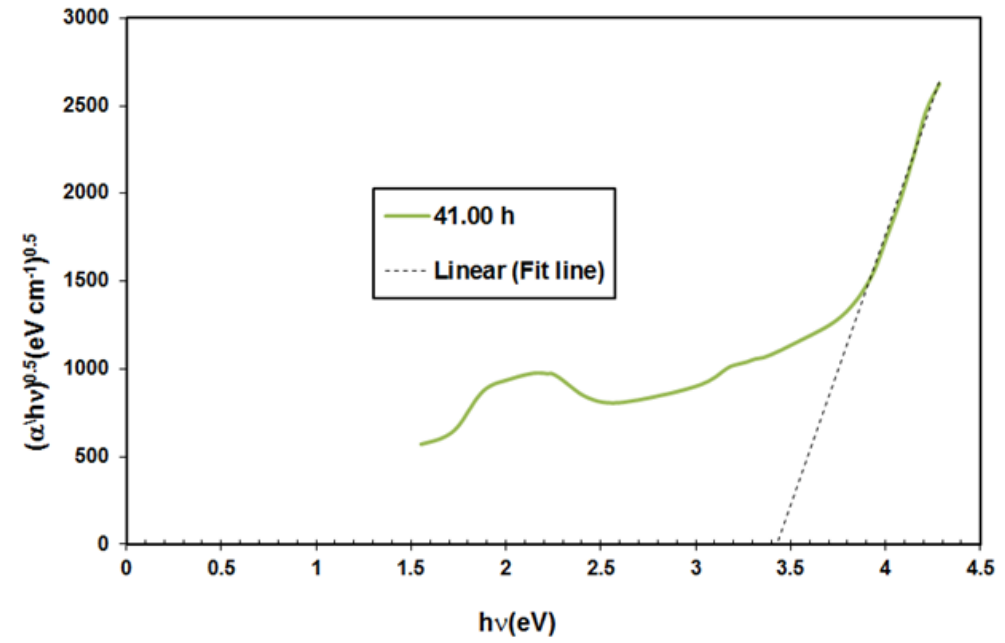

Fig. 5. Tauc's plot corresponding to indirect band gap for irradiated PANI samples at alpha particle irradiation time $41.00 \mathrm{~h}$

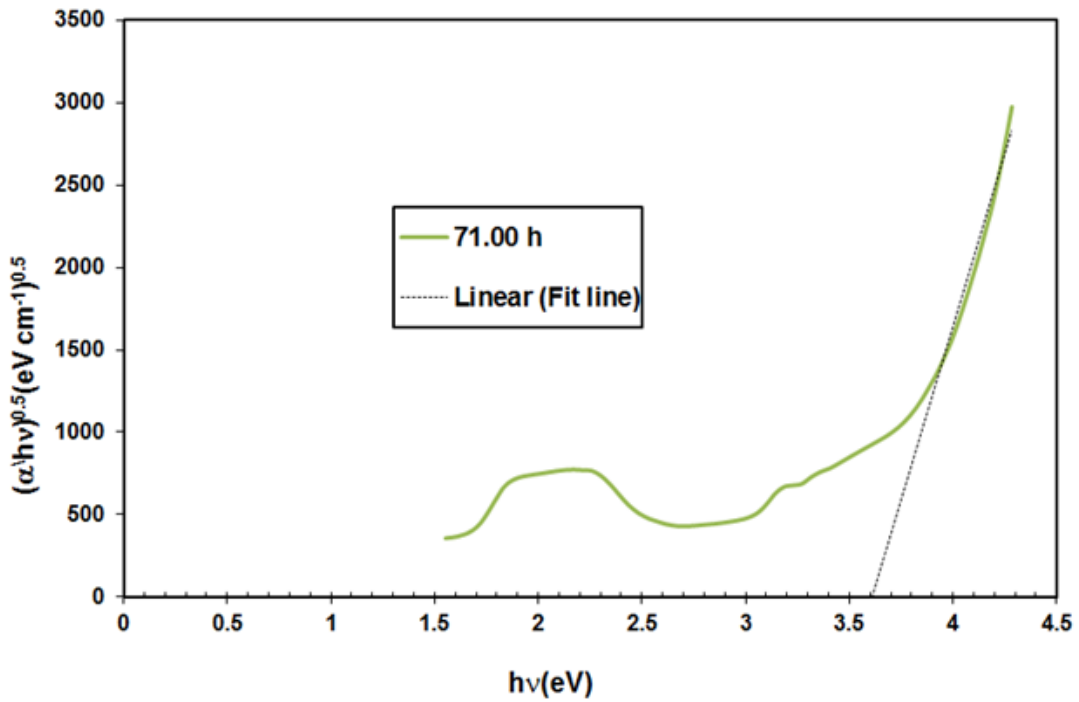

Fig. 6. Tauc's plot corresponding to indirect band gap for irradiated PANI samples at alpha particle irradiation time $71.00 \mathrm{~h}$. 
Table I: Variations of the indirect band gap and cluster size for alpha particle virgin and irradiated PANI samples at different irradiation times and fluences

\begin{tabular}{lllr}
\hline Sample & Fluence $\left(\right.$ alpha $\left./ \mathrm{cm}^{2}\right)$ & $\begin{array}{l}\text { Indirect } \\
\text { band gap } \\
\text { energy }(\mathrm{eV})\end{array}$ & Cluster size \\
\hline Virgin & 0 & 3.60 & 91 \\
$6.50 \mathrm{~h}$ & $1.16 \times 10^{8}$ & 3.50 & 96 \\
$41.00 \mathrm{~h}$ & $7.48 \times 10^{8}$ & 3.42 & 101 \\
$71.00 \mathrm{~h}$ & $1.20 \times 10^{9}$ & 3.49 & 97 \\
\hline
\end{tabular}

\subsection{Cluster size}

The relation between the band gap $\left(\mathrm{E}_{\mathrm{g}}\right)$ and the cluster size (number of carbon atoms per cluster), $\mathrm{N}$, can be determined according to the following formula [23]:

$$
E_{g}=\frac{34.3}{\sqrt{N}} \mathrm{eV}
$$

The calculated values of $\mathrm{N}$ corresponding to the fluence for the virgin and irradiated PANI samples were displayed in Table I. From the Table I one can find that the cluster size for virgin and irradiated PANI varies from 91 to 101 carbon atoms. The maximum value for cluster size was found at irradiation time $41.00 \mathrm{~h}$ whereas the minimum value for the band gap energy. This result confirms appearing of clusters in the morphology of the PANI sample at $41.00 \mathrm{~h}$ irradiation time as shown in Fig. 1. Carbon enriched domains created in irradiated PANI samples may be responsible for the decreasing in band gap.

\section{Conclusions}

The morphology and optical properties of PANI thin film polymer in nanostructure which irradiated with alpha particle at different fluences $\left(1.16 \times 10^{8}-1.20 \times 10^{9}\right.$ alphas $\left./ \mathrm{cm}^{2}\right)$ with energy $5.32 \pm 0.23 \mathrm{MeV}$ were investigated. The radiation damage is almost regular along the track of alpha particles in PANI samples. The SEM images of irradiated PANI samples, showed the highest number of carbon atoms per cluster were found at irradiation time $41.00 \mathrm{~h}$. The results of the optical properties showed that the samples after irradiations with the present alpha particle fluences are sensitive to the visible light at the wavelengths $530-705 \mathrm{~nm}$. The $\mathrm{E}_{\mathrm{g}}-$ value was reduced due to alpha particle irradiations. This may be attributed to the formation of a conjugated system of bonds in PANI films due to bond cleavage by radiation. Also, Carbon enriched domains created in irradiated PANI samples may be responsible for the decreasing in band gap.

\section{Acknowledgment}

The authors are very thankful to members of the Physics Department, Beni -Suef University, Egypt, for their continuous encouragement and valuable support.

\section{References}

[1] Kim, B., Koncar, V. and Devaux, E., Electrical properties of conductive polymers: PET - nanocomposites' fibers, AUTEX Research Journal 4(2004) 9-13.

[2] Park, J. K. and Kwon, O-P., Enhanced electrical conductivity of polyaniline film by a low magnetic field, Synth. Met. 160 (2010) 728-731.

[3] Gerard, M., Chaubey, A. and Malhotra, B. D., Application of conducting polymers to biosensors, Biosensor Bioeleton., 17 (2002) 345-359.

[4] Smith, J. D. S., Intrinsically Electrically Conducting Polymers. Synthesis, Characterization and their Applications, Prog. Polym. Sci., 23 (1998) 57-79.

[5] Wessling, B., From conductive polymers to organic metals, Chem. Innov., 31 (2001) 34-40.

[6] Cho, Y. H., Cho, M.S., Choi, H. J., and Jhon, M. S., Electrorheological characterization of polyaniline-coated poly(methyl methacrylate)suspensions, Colloid Polym. Sci., 280 (2002) 1062-1066.

[7] MacDiarmid, A. G., Synthetic Metals": A Novel Role for Organic Polymers (Nobel Lecture), Angew. Chem. Int. Ed. 40 (2001) 2581-2590.

[8] Stejskal, J. and Gilbert, R. G., Polyaniline preparation of conducting polymer. Pure Appl. Chem., 74 (2002) 857-867.

[9] Choi, H. J., Cho, M. S., Kim, J.W., Kim, C.A., and Jhon, M. S., A yield stress scaling function for electrorheological fluids, Appl. Phys. Lett., 78 (2001) 3806-3809.

[10] Durrani, S.A. and Bull, R.K.., Solid State Nuclear Track Detection: Principles, Methods and Applications, Pergamon Press, Oxford, (1987)

[11] Lee, E.H., ion-beam modification of polymeric materials- fundamental, principles and applications. Nucl. Instrum. Methods B, 151 (1999) 29-41.

[12] IAEA-TECDOC-1617, Controlling of Degradation Effects in Radiation Processing of Polymers, May 2009, Vienna, Austria.

[13] Yates, B. W. and Shinozaki, D.M. Radiation degradation of poly (methyl methacrylate) in the soft x-ray region, J. Polym. Sci., 31 (1993) 1779- 1784.

[14] Kudoh, H., Sasuga, T., Seguchi, T. and Katsumura, Y., High-energy-ion-irradiation effects on polymer materials: 3. The sensitivity of cellulose triacetate and poly (methyl methacrylate), Polymer 37 (1996) 2903- 2908.

[15] Lee, E.H., Rao, G.R. and Mansur, L. K., Hardness Enhancement and Crosslinking Mechanisms in Polystyrene Irradiated with High Energy Ion-Beams, Materials Science Forum 248-249 (1997) 135-146. 
[16] Eissa, M. F., Study the effect of post-irradiation gamma ray doses on optical and spectral response of CR-39 polymer track recorder, Int. J. Low Radiat. 8 (2011)10-19.

[17] Eissa, M. F., M. A. kaid, M. A. and Kamel, N. A., Study of the effects of low and high linear energy transfers on Poly(methylmethacrylate) samples, Journal of applied polymer Science, 125(2012) 3682-3687.

[18] Singh S. and Prasher S. A., comparison of modifications induced by $\mathrm{Li}^{3+}$ and $\mathrm{O}^{6+}$ ion beam to Makrofol-KG and CR-39 polymeric track detectors, Nucl. Instr. Meth. B, 244 (2006)252-256.

[19] Enge, W., Introduction to plastic nuclear track detectors, Nuclear track, 4 (1980) 283-308.

[20] Ziegler, J. F. SRIM-2013, available online at: www.SRIM.org.

[21] Halary, J. L., F. Laupretre, F. and Monnerie, L., Polymer Materials; Macroscopic Properties and Molecular Interpretations, Wiley, 2013, ISBN: 9780470922880.

[22] Negi, A., Semwal, A., Chandra, S., Hariwal, R.V., Sonkawade, R.G., Kanjilal, D., Rana, J.M.S. and Ramola,R. C., Modifications induced by $\mathrm{Li}^{+3}, \mathrm{Ni}^{+9}$ and $\mathrm{Au}^{+9}$ ion beams to CR-39 polymer track detector, Radiation Measurements 46 (2011) 127-132.

[23] Fink, D., Chung, W. H., Klett, R., Schmoidt, A., Cardosa, J., Montiel, R., Vazqez, M.H., Wang, L., Hosoi, F., Omichi, H.and Goppelt-Langer, P., Carbonaceous clusters in irradiated polymers as revealed by UV-visible spectrometry, Radiat. Eff. Def. Solids 133(1995) 193-208. 